\title{
A new analgesic method, two-minute sciatic nerve press, for immediate pain relief: a randomized trial Jiman $\mathrm{He}^{* 1}$, Bin $\mathrm{Wu}^{\dagger 2}$, Xianrong Jiang ${ }^{\dagger 3}$, Fenglin Zhang ${ }^{\dagger 4}$, Tao $\mathrm{Zhao}^{5}$ and Wenlon Zhang 6
}

\begin{abstract}
Address: 'Biomedicine (TC), Chinese Academy of Sciences, Beijing, 100080 China \& Rhode Island Hospital, Brown University, RI 02903, USA, ${ }^{2}$ Renal Department, Anhui Province Hospital, Anhui Medical University, Hefei, 230001, China, ${ }^{3}$ Department of Biology, Hefei Teachers College, Hefei, 230061, China, ${ }^{4}$ Department of Oncology, Maanshan People Hospital, Maanshan, 243000, China, ${ }^{5}$ Department of Emergency, Anhui Province Hospital, Hefei, 230001, China and ' Department of Dentistry, Chuzou Zhongxiyi Hospital, Chuzou, 239000, China
\end{abstract}

Email: Jiman He* - jiman_he@brown.edu; Bin Wu - wubin@ahmu.edu.cn; Xianrong Jiang - hxh5639@sina.com; Fenglin Zhang - zhangfenglin@csco.org.cn; Tao Zhao - zhaotaoyin@163.com; Wenlon Zhang - wenlonzhang@sohu.com

* Corresponding author †Equal contributors

Published: 25 January 2008

BMC Anesthesiology 2008, 8: I doi:10.1 I86/147|-2253-8-1
Received: 26 June 2007

Accepted: 25 January 2008

This article is available from: http://www.biomedcentral.com/I47/-2253/8/I

(c) 2008 He et al; licensee BioMed Central Ltd.

This is an Open Access article distributed under the terms of the Creative Commons Attribution License (http://creativecommons.org/licenses/by/2.0), which permits unrestricted use, distribution, and reproduction in any medium, provided the original work is properly cited.

\begin{abstract}
Background: Current analgesics have drawbacks such as delays in acquisition, lag-times for effect, and side effects. We recently presented a preliminary report of a new analgesic method involving a two-minute sciatic nerve press, which resulted in immediate short-term relief of pain associated with dental and renal diseases. The present study investigated whether this technique was effective for pain associated with other disease types, and whether the relief was effective for up to one hour.
\end{abstract}

Methods: This randomized, placebo-controlled, parallel-group trial was conducted in four hospitals in Anhui Province, China. Patients with pain were sequentially recruited by participating physicians during clinic visits, and 135 patients aged $15-80$ years were enrolled. Dental disease patients included those with acute pulpitis and periapical abscesses. Renal disease patients included those with kidney infections and/or stones. Tumor patients included those with nose, breast, stomach and liver cancers, while Emergency Room patients had various pathologies. Patients were randomly assigned to receive a "sciatic nerve press" in which pressure was applied simultaneously to the sciatic nerves at the back of both thighs, or a "placebo press" in which pressure was applied to a parallel region on the front of the thighs. Each fist applied a pressure of $\mathrm{II}-20 \mathrm{~kg}$ for 2 minutes. Patients rated their level of pain before and after the procedure.

Results: The "sciatic nerve press" produced immediate relief of pain in all patient groups. Emergency patients reported a $43.5 \%$ reduction in pain $(p<0.00 \mathrm{I})$. Significant pain relief for dental, renal and tumor patients lasted for 60 minutes $(p<0.001)$. The peak pain relief occurred at the 10 $-20^{\text {th }}$ minutes, and the relief decreased $47 \%$ by the $60^{\text {th }}$ minutes.

Conclusion: Two minutes of pressure on both sciatic nerves produced immediate significant short-term conduction analgesia. This technique is a convenient, safe and powerful method for the short-term treatment of clinical pain associated with a diverse range of pathologies.

Trial registration: Current Controlled Trials ACTRNOI 2606000439549 


\section{Background}

Human beings have long suffered from pain caused by diseases. In medicine, pain is one of the most common reasons for patients to seek care. Analgesics used in current practice have drawbacks such as delays in acquisition and lag-times for effect after administration. In addition, many commonly used analgesics have considerable sideeffects [1-4].

Many non-drug analgesic interventions have been used to help manage pain, including acupuncture, cryoanalgesia, transcutaneous electrical nerve stimulation (TENS), exercise, and music therapy, etc. [5-7]. Though literature reviews document the efficacy of some non-drug analgesic interventions $[5,8,9]$, their use are usually restricted to some pain centers, and, the clinical effectiveness of some analgesic methods are controversial [10-12].

We recently published a preliminary report on a new analgesic method, a 2-min sciatic nerve press, which immediately relieved pain brought on by various dental and renal diseases [13]. The technique is simple, can be used any time, any place, immediately upon the onset of pain (including outside of a hospital setting). No side effects have yet been seen.

The present study examined whether the technique worked on more pathologies, and whether pain relief extended to up to one hour.

\section{Methods}

\section{Setting and procedures}

The study was a randomized, single-blinded, placebocontrolled, parallel-group trial consisting of 135 patients treated between October 2005 and May 2006 at four hospitals in China. The study was separately approved by each participating hospital - Anhui Province Hospital, Hefei, 230001, China (Approval date-June 2, 2005); Maanshan People Hospital, Maanshan, 243000, China (Approval date-May 20, 2005); Tongling Count Hospital, Tongling Count, 244100, China (approval date-October 18, 2005), and Chuzou Zhongxiyi Hospital, Chuzou, 239000, China (approval date-May 28, 2005). Written informed consent was obtained from each participating patient.

Patients were divided into "sciatic press" and "placebo press" groups. Instructions and explanations were equally provided to all patients. Patients were informed that the experiments were designed to test whether the methods were effective for pain relief. Patients were advised that they could discontinue participation in the study at any time without penalty, and that their healthcare treatment would not otherwise be affected.
After informed consent was obtained, the participating doctor(s) or assistants taught the patients how to evaluate pain using a visual analogue scale (VAS), where pain scored from " 0 " for no pain, to " 10 " for most pain. Thereafter, randomization of patients to the "sciatic press" or "placebo press" group was performed using the method of Random Permuted Blocks. The three stages of the test were then described to each patient: baseline pain rating, 2 min of leg pressure while lying down, and post-pressure pain rating at the specified times.

Two test designs were used. For Emergency patients, pain was rated once after pressure application. In contrast, renal, dental and tumor patients underwent the $60 \mathrm{~min}$ test in which pain was rated at 10-min intervals for a period of one hour after pressure application. In this context, the ' 0 min' point indicated the time at which the 2min leg pressure stopped and the post-pressure pain rating stage commenced. The baseline pain rating and leg pressure stages were identical for the two types of tests.

The positions of sciatic nerve pressure and the fist gesture used for pressure application are shown in Figure 1. This manipulation technique has been previously described in detail [13]. Briefly, 2 min of pressure with the dorsal, proximal phalangeal surface of the fists (not the knuckles or finger tips) was applied simultaneously to the sciatic nerve sites or the placebo location on both legs. For the "sciatic nerve press", 11 to $20 \mathrm{~kg}$ of pressure was applied to the sciatic nerves on the back of the thighs with each fist, while patients lay prone. For the "placebo press", the same pressure was applied to a parallel spot on the front of the thighs, while patients lay supine. Patients then stood and rated their pain using a visual analogue scale table. The amount of force applied within the range of 11-20 kg depended on the patient body type, with heavily muscled large-bodied patients receiving higher pressures
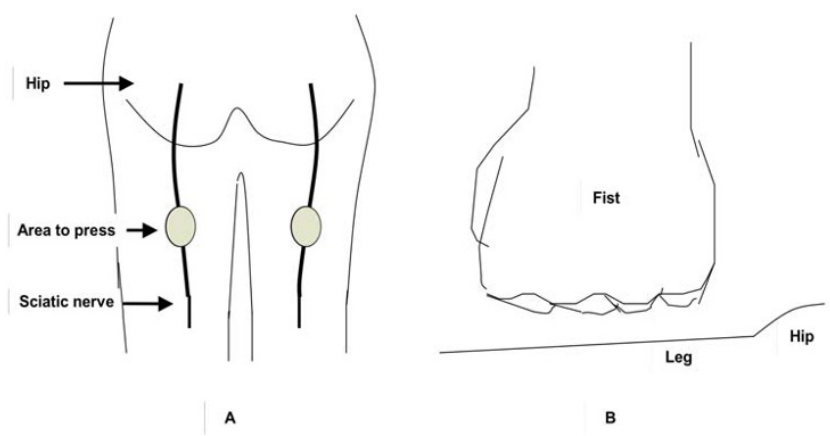

Figure I

(A) The location of the pressure areas on the sciatic nerve.

(B) The gesture for applying fist pressure. 
than thin patients. Doctors learned the pressure force applied by repeatedly pressing on a weighted balance.

\section{Participants}

The inclusion criterion was any patient who was feeling pain during a clinic visit to the Emergency room, Renal, Dental, or Oncology Clinics. Exclusion criteria were age less than 15 years, emotional instability, or administration of another analgesic within 12 hours of the test. For enrolled patients, the dental diseases included acute pulpitis and periapical abscesses. The renal diseases included infections and/or stones, and the tumors included those of the nose, breast, stomach, and liver. While Emergency Room enrollees had various pathologies. No patients had previously been treated with the sciatic press method. For tumor patients, analgesics were discontinued 11-13 hours prior to the test, and the study began when patients reported they had moderate-to-severe pain.

The patient groups and characteristics are shown in Table 1. Of the 229 solicited patients, 57 refused to join and 37 were considered ineligible, leaving 135 patients aged from $15-80$ years to participate in the study. Of those 135 , two renal patients in the placebo group withdrew in the middle of the 60 min test stating that the pain was too severe to continue.

\section{Statistical analysis}

Renal, dental and oncology patients were analyzed as one group in terms of relief of pain. Emergency room patients were analyzed separately, and without pathology categorization. The baseline VAS scores and age of the participants were compared between the "sciatic press" and "placebo press" groups using t-tests. Categorical data were analyzed using chi-square, or Fisher Exact tests. In terms of pain relief, changes from baseline were assessed using paired $t$-tests for both groups. Comparisons with the "pla- cebo press" groups were performed using an analysis of covariance procedure, with adjustment for baseline VAS score, gender and age. All tests were two-sided, and a pvalue of $<0.05$ was considered to indicate significance. All statistical analyses were performed using SPSS statistical software (release 13.0).

\section{Results}

The study included 95 Emergency Department patients with various pain-related conditions (Figure 2). Following application of the "sciatic press" technique, patients recorded a $43.5 \%$ decrease in the mean VAS pain scores ( $\mathrm{p}$ $<0.001)$. The pain relief was significantly greater than with the "placebo press" ( $p<0.001)$. Notably, over $70 \%$ of Emergency patients reported pain relief after the "sciatic press".

To test the longer-term effectiveness of the "sciatic press" method, 40 patients with pain from tumors, dental and renal diseases were tested for a period of one hour (Figure 3). Pain data for the three patient groups were pooled, and demonstrated that mean VAS scores were lower at all time points $(\mathrm{p}<0.001)$ for the "sciatic press" group compared to the placebo group (Figure $3 \mathrm{~A}$ ). Pain was reduced by $54.5 \%$ at the $10^{\text {th }}$ minute, and by $35.3 \%$ at the $60^{\text {th }}$ minute.

In the "placebo press" group, the VAS score was reduced by $11-15 \%$ within the first $10 \mathrm{~min}$, and by $23.4-29.5 \%$ between the $40^{\text {th }}$ and $60^{\text {th }}$ minutes. Data were also expressed by subtracting "placebo press" values from "sciatic press" values and plotting the resultant value against time (Figure 3B). The analysis showed that the peak pain relief of this technique occurred at $10^{\text {th }}-20^{\text {th }}$ minutes, with relief reduced $47 \%$ by the $60^{\text {th }}$ minute.

Table I: Patients groups and characteristics at inclusion

\begin{tabular}{|c|c|c|c|}
\hline & Placebo press $(n=70)$ & Sciatic press $(n=65)$ & p value \\
\hline \multicolumn{4}{|l|}{ Emergency test } \\
\hline Participants (n) & 49 & 46 & - \\
\hline Male (\%) & $57.1 \%$ & $67.3 \%$ & 0.24 \\
\hline Age & $41.9 \pm 13.4$ & $37.6 \pm 12.7$ & 0.12 \\
\hline Baseline VAS & $7.1 \pm 1.88$ & $7.3 \pm 1.63$ & 0.49 \\
\hline \multicolumn{4}{|c|}{60 min test in renal, dental and tumor patients } \\
\hline Participants (n) & 21 & 19 & - \\
\hline Renal & $28.6 \%$ & $21.1 \%$ & 0.86 \\
\hline Dental & $38.1 \%$ & $42.1 \%$ & \\
\hline Male (\%) & $42.9 \%$ & $52.6 \%$ & 0.54 \\
\hline Age & $42.5 \pm 18.3$ & $41.6 \pm 15.4$ & 0.88 \\
\hline Baseline VAS & $6.05 \pm 1.36$ & $6.39 \pm 1.55$ & 0.46 \\
\hline
\end{tabular}

Age, gender and baseline VAS: mean ( \pm SD), t-test. 


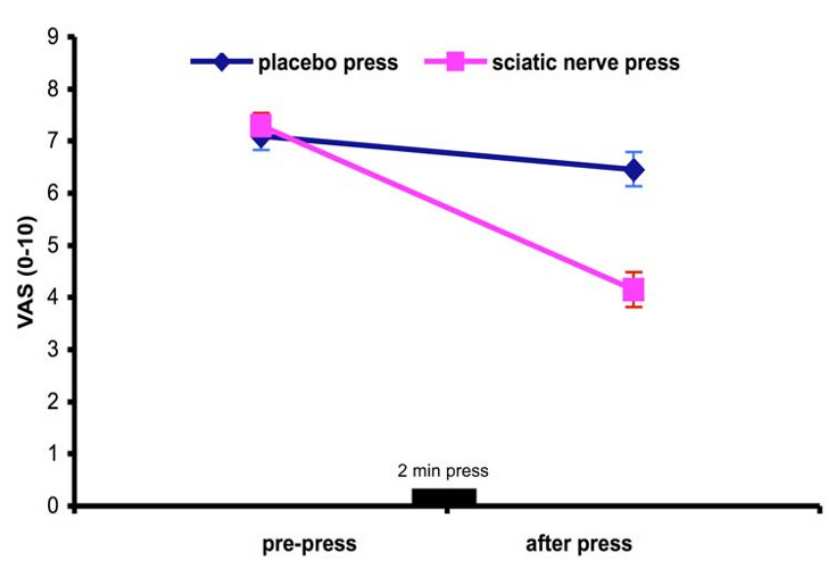

Figure 2

Pain relief in emergency patients. Data represent the mean VAS score $( \pm$ SE) $(p<0.00 \mathrm{I})$.

\section{Discussion}

Mechanical pressure can cause nerve abnormalities. A number of studies have reported the effect of pressure on nerves using different force levels and time periods of up to several weeks [14-21]. In animal models, pressure studies usually involve surgically exposed nerves $[16,17,22]$. However, the present method uses a much shorter time period, two minutes for the pressure on the intact skin. Thus far, the method has been applied to over 600 subjects across 10 hospitals and universities, and there have been no reports of adverse side-effects. The type of acute pressure applied in this technique $(11-20 \mathrm{~kg}$ by each fist $(150-380 \mathrm{mmHg}$ ) for up to $2 \mathrm{~min}$ ) is not uncommon in daily activities, especially for people involved in sports or heavy manual labor. However, nerve injuries as a result of this procedure remain a possibility, and must be taken into account in future studies. The present technique does not involve chronic pressure on nerves, of which even a very small amount could cause severe nerve dysfunction. For example, chronic pressure on the sciatic nerve by internal tension of the obturator muscle or through anatomical abnormalities in the piriformis muscle could cause pain [23-26]. Surgery to relieve the muscle pressure results in immediate pain relief [23,26-28].

The present study was single-blinded rather than doubleblinded as the method is highly effective for pain relief, and doctors could easily identify the placebo or the active treatment during the test. After patients reported pain scores, patients themselves recorded their pain assessments on a VAS table, or in some cases, had the doctors record the VAS score. However, it would be a stronger study to have employed a blinded observer, which we will include in our future studies.

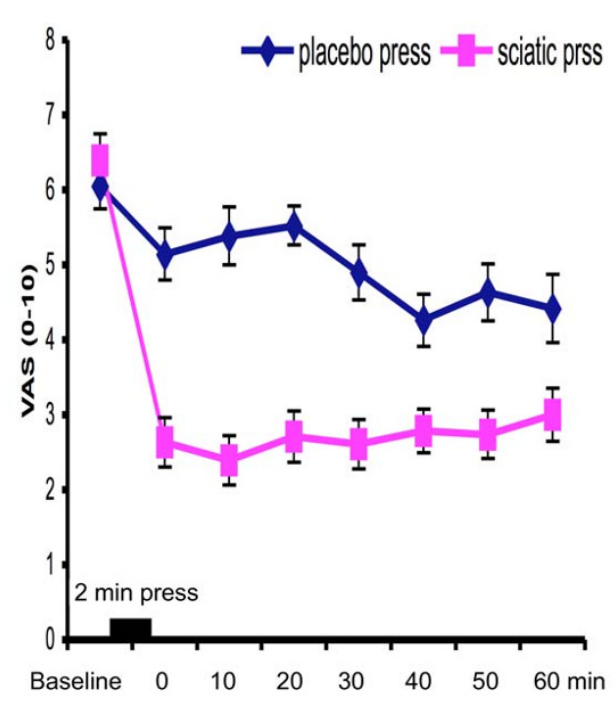

A

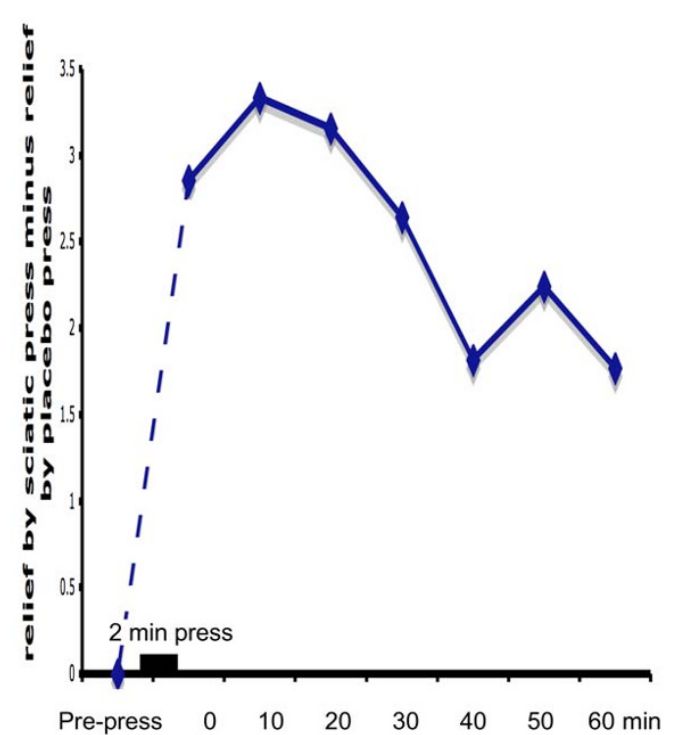

B

\section{Figure 3}

The 60 min test for the combined group of renal, dental and oncology patients. Data represent the mean VAS score ( \pm SE). ( $P$ $<0.001$ for all post-pressure points between the "sciatic press" and placebo groups). 
To our knowledge, no clinical studies have reported on the use of pressure stimulation of the anterior or posterior thigh area for pain relief. The study design ensured no patients had previously undergone this technique, and we believe the 'blinding' procedures remained secure during testing. The pressure of $11-20 \mathrm{~kg}$ with each fist was chosen based on pilot studies using Chinese patients. Such a pressure range may differ for Western populations given the difference in average body size.

The present study showed that Emergency patients had a mean baseline VAS score of 7.2, and that the "sciatic press" resulted in a $43.5 \%$ reduction in pain. Nearly $30 \%$ of these patients reported no pain relief. Similarly, our previous study showed that renal patients had a mean baseline VAS score of 7.7, that the sciatic press method resulted in a $52.2 \%$ pain reduction, and that $40 \%$ of renal patients felt no pain relief [13]. The previous study also showed that dental patients had a baseline VAS score of 6.4 , and that the treatment resulted in a $66.4 \%$ reduction in pain [13], and these are similar results to those from tumor patients who had a baseline VAS score of 5.8 and pain reduction of $70.7 \%$ (tumor patient test data are not shown in the present report). These data suggest that the analgesic effect may correlate with the baseline pain score. However, the present study was not comprehensive enough to make such a statistical connection, and this hypothesis awaits further testing in future larger studies.

In the "placebo press" group undergoing the 60 min test, VAS scores decreased markedly in later time periods (23.4-29.5\% between the 40th and 60th min) compared to the first $10 \mathrm{~min}$ (11-15\% reduction). In this group, $25 \%$ of patients had pain at the early time points but not at later times. For this study setting, we cannot rule out the total or partial contribution of a placebo effect, or some anterior nerve stimulation to the late drop. Pain discontinuity could also be contributing partially.

Stimulation of peripheral nerves elevates the pain threshold [29-32]. According to the Gate Control Theory of Pain [33], stimulation of large-diameter afferent fibers can inhibit the transmission of nociceptive information from the dorsal horn to higher brain centers. This inhibition occurs rapidly and is thought to involve the wide dynamic range (WDR) neurons [34-36]. The resulting analgesic effect is considered to be a short-lasting, segmental inhibition of pain [37-39]. This theory may explain the rapid pain relief observed in some situations. However, the pain relief created by the present method is not limited to the segmental level, but occurs throughout the body. Also, significant pain relief lasted for 60 minutes. These observations suggest possible activation of multiple inhibitory systems. Another mechanism possibly involved is activation of the endogenous opioid system. Yao found that low frequency stimulation of the rat sciatic nerve increased the pain threshold by $50 \%$, and that this effect was antagonized by Naloxone which suggested activation of the opioid system [40].

Pinch press of the rat sciatic nerve with a vascular clip (pinch force $120 \mathrm{~g}$ ) caused attenuation of WDR neuron responses to various innocuous and noxious stimuli [41]. While the WDR neuron response to superficial peroneal nerve stimulation was shown to increase in cats when the sciatic nerve was under a clip pressure with a pinch force $180 \mathrm{~g}$, the response was inhibited when using low frequency stimulation $(0.2 \mathrm{~Hz})$ [32]. In the present clinical study, despite it being likely that there were slight variations in the pressure location and pressure force applied, pain relief was consistently achieved in all test groups. The current findings are similar to those from our pilot studies in which mechanical pressure was applied at different locations along the sciatic nerves using methods other than fists (data not shown).

\section{Conclusion}

Two minutes of pressure on both sciatic nerves can produce immediate and significant conduction analgesia. This procedure provides a convenient, safe and powerful method for the short-term treatment of clinical pain induced by diverse disease types.

\section{Competing interests}

The author(s) declare that they have no competing interests.

\section{Authors' contributions}

$\mathrm{JH}$ was the primary investigator, had full access to all data, and takes responsibility for the integrity of the data and the decision to submit the work for publication. BW participated in renal clinical tests and data analysis. XJ collaborated in the pilot study and the mechanism study. FZ collaborated in the technical aspects and in administrative support for tumor patient testing. TZ participated in Emergency room tests. WZ collaborated in clinical tests on dental patients and in data interpretation. All authors read and approved the manuscript.

\section{Acknowledgements}

We thank: Jack R. Wands, MD of Brown University, and Robert Dowman, $\mathrm{PhD}$ of Clarkson University for their important help with the study; Gail Donaldson, MD, of Buffalo, NY, and Rolf Carlson, MD of Rhode Island Hospital, Brown University for their editorial assistance, and, Yong Du, PhD of Robert Koch-Institute, Germany, for the help in data analysis. We thank Pingniu Wei, BS, Anqing Normal College, China, Huadong Lu, MD, Jingxu Wang, MD, and Rongge Cao, MD, Anhui Province Hospital, China, for their assistance in tests on ER patients. Thanks go to: Shaoshan Wu, MD and Hao Chen, MD, Anhui Province Hospital, China, for their assistance in clinical tests on renal patients, and to Lin Liu, MD, and Yao Ma, BS, Maanshan People's Hospital, China, for their assistance in clinical tests on tumor patients. We also thank Sigang Yang, DDS, Tongling County Hospital, China, Weihua 
Dong, DDS, Weihua Dental Clinics, Niubu, Wuwei, China and, Yunxia Huang, MD, Chuzou Hospital, China, for their assistance in clinical tests on dental patients.

\section{References}

I. Davis MP, Srivastava M: Demographics, assessment and management of pain in the elderly. Drugs Aging 2003, 20(I):23-57.

2. Stiel D: Exploring the link between gastrointestinal complications and over-the-counter analgesics: current issues and considerations. Am J Ther 2000, 7(2):91-98.

3. Forman WB: Opioid analgesic drugs in the elderly. Clin Geriatr Med 1996, I 2(3):489-500.

4. Hersh EV, Moore PA, Ross GL: Over-the-counter analgesics and antipyretics: a critical assessment. Clin Ther 2000, 22(5):500-548.

5. Wright A, Sluka KA: Nonpharmacological treatments for musculoskeletal pain. Clin J Pain 200I, I7(I):33-46.

6. Rakel B, Barr JO: Physical modalities in chronic pain management. Nurs Clin North Am 2003, 38(3):477-494.

7. Rusy LM, Weisman SJ: Complementary therapies for acute pediatric pain management. Pediatr Clin North Am 2000, 47(3):589-599.

8. Trescot AM: Cryoanalgesia in interventional pain management. Pain Physician 2003, 6(3):345-360.

9. Nadler SF: Nonpharmacologic management of pain. J Am Osteopath Assoc 2004, 104(Suppl 8):S6-12.

10. Ernst E: Acupuncture--a critical analysis. J Intern Med 2006, 259(2): 125-137.

II. Milne S, Welch V, Brosseau L, Saginur M, Shea B, Tugwell P, Wells G: Transcutaneous electrical nerve stimulation (TENS) for chronic low back pain. Cochrane Database Syst Rev 200I:CD003008.

12. Carroll D, Moore RA, McQuay HJ, Fairman F, Tramer M, Leijon G: Transcutaneous electrical nerve stimulation (TENS) for chronic pain. Cochrane Database Syst Rev 200I:CD003222.

13. He J, Wu B, Zhang W, Ten G: Immediate and short-term pain relief by acute sciatic nerve press: a randomized controlled trial. BMC Anesthesiol 2007, 7:4.

14. Macgregor RJ, Sharpless SK, Luttges MW: A pressure vessel model for nerve compression. J Neurol Sci 1975, 24(3):299-304.

15. Lundborg G DLB: Pathophysiology of nerve compression. In Nerve Compression Syndromes: Diagnosis and treatment, Chapter 3. Edited by: Szabo RM. Slack Inc, Thorofare, NJ .

16. Dahlin LB, Shyu BC, Danielsen N, Andersson SA: Effects of nerve compression or ischaemia on conduction properties of myelinated and non-myelinated nerve fibres. An experimental study in the rabbit common peroneal nerve. Acta Physiol Scand 1989, I36(I):97-105.

17. Fern R, Harrison PJ: The contribution of ischaemia and deformation to the conduction block generated by compression of the cat sciatic nerve. Exp Physiol 1994, 79(4):583-592.

18. Lundborg G, Myers R, Powell H: Nerve compression injury and increased endoneurial fluid pressure: a "miniature compartment syndrome". I Neurol Neurosurg Psychiatry 1983, 46(I2): III19-II 24.

19. Ochoa J, Fowler TJ, Gilliatt RW: Anatomical changes in peripheral nerves compressed by a pneumatic tourniquet. J Anat 1972, I I 3(Pt 3):433-455.

20. Powell HC, Myers RR: Pathology of experimental nerve compression. Lab Invest 1986, 55(I):91-100.

21. Gelberman RH, Szabo RM, Williamson RV, Dimick MP: Sensibility testing in peripheral-nerve compression syndromes. An experimental study in humans. J Bone Joint Surg Am 1983, 65(5):632-638.

22. Sarikcioglu L, Yaba A, Tanriover G, Demirtop A, Demir N, Ozkan O: Effect of severe crush injury on axonal regeneration: a functional and ultrastructural study. J Reconstr Microsurg 2007, 23(3): 143-149.

23. Benson ER, Schutzer SF: Posttraumatic piriformis syndrome: diagnosis and results of operative treatment. J Bone Joint Surg Am 1999, 8 I(7):94I-949.

24. Solheim LF, Siewers $P$, Paus $B$ : The piriformis muscle syndrome. Sciatic nerve entrapment treated with section of the piriformis muscle. Acta Orthop Scand 198I, 52(I):73-75.
25. Mullin $\mathrm{V}$, de Rosayro M: Caudal steroid injection for treatment of piriformis syndrome. Anesth Analg 1990, 7 I (6):705-707.

26. Meknas $K$, Christensen $A$, Johansen $O$ : The internal obturator muscle may cause sciatic pain. Pain 2003, 104(I-2):375-380.

27. Chen WS: Bipartite piriformis muscle: an unusual cause of sciatic nerve entrapment. Pain 1994, 58(2):269-272.

28. Sayson SC, Ducey JP, Maybrey JB, Wesley RL, Vermilion D: Sciatic entrapment neuropathy associated with an anomalous piriformis muscle. Pain 1994, 59(I): I49-152.

29. Woolf CJ, Mitchell D, Barrett GD: Antinociceptive effect of peripheral segmental electrical stimulation in the rat. Pain 1980, 8(2):237-252.

30. Chung JM, Lee KH, Hori Y, Endo K, Willis WD: Factors influencing peripheral nerve stimulation produced inhibition of primate spinothalamic tract cells. Pain 1984, 19(3):277-293.

31 . Jorum $E$ : The analgesic effect of peripheral nerve stimulation in various tests of nociception in rats. Acta Physiol Scand 1988, 133(2): $13 \mid-138$.

32. Hanai $F$ : Effect of electrical stimulation of peripheral nerves on neuropathic pain. Spine 2000, 25(15): $1886-1892$.

33. Melzack R, Wall PD: Pain mechanisms: a new theory. Science 1965, 150(699):97I-979.

34. Cohen ML, Arroyo JF, Champion GD, Browne CD: In search of the pathogenesis of refractory cervicobrachial pain syndrome. A deconstruction of the RSI phenomenon. Med J Aust 1992, I 56(6):432-436.

35. Wall PD: Dorsal horn electrophysiology. In: Iggo A (Ed), Handbook of Sensory Physiology-Somatosensory System. Berlin: Springer-Verlag. 1973:253-270.

36. Price DD: Psychological and neural mechanisms of pain. New York, Raven. 1988.

37. Andersson SA: Pain control by sensory stimulation. In Advances in Pain Research and Therapy, Volume 3. Edited by: Bonica JJ, Lieberskiend JC, Albe-Fessard DG. New York: Raven Press. 1979:569-585.

38. Garrison DW, Foreman RD: Decreased activity of spontaneous and noxiously evoked dorsal horn cells during transcutaneous electrical nerve stimulation (TENS). Pain 1994, 58(3):309-315.

39. Hollman JE, Morgan BJ: Effect of transcutaneous electrical nerve stimulation on the pressor response to static handgrip exercise. Phys Ther 1997, 77(1):28-36.

40. Yao T, Andersson S, Thoren P: Long-lasting cardiovascular depressor response following sciatic stimulation in spontaneously hypertensive rats. Evidence for the involvement of central endorphin and serotonin systems. Brain Res 1982, 244(2):295-303.

41. Kawasaki M, Ushida T, Tani T, Yamamoto H: Changes of wide dynamic range neuronal responses to mechanical cutaneous stimuli following acute compression of the rat sciatic nerve. J Orthop Sci 2002, 7(1): I II-116.

\section{Pre-publication history}

The pre-publication history for this paper can be accessed here:

http://www.biomedcentral.com/1471-2253/8/1/prepub 